\title{
Does Environmental Information Disclosure Increase the Firm Value and Investors' Confidence?
}

\author{
Tangmei Yuan ${ }^{1, a^{*}}$ \\ ${ }^{1}$ Department of Accounting, Linyi University, Shandong, China \\ ayyytm@126.coml
}

Keywords: Environmental information disclosure; Firm value; Investors' confidence

\begin{abstract}
Use all A-share listed companies of main board market in 2012 as samples, this paper investigate the influence of environmental information disclosure behavior on the firm future market value and investors` confidence in capital market. The research conclusion indicate that: (1) The listed companies` environmental information disclosure behavior index and environmental information disclosure quality index are both significantly positive correlation with the company's next seasonal added value; (2) The disclosure quality significantly positive correlation with investor confidence. In short, the high quality of disclosure of environmental information can significantly improve the company value and investors' confidence in Chinese capital market.
\end{abstract}

\section{Introduction}

Enterprise is the direct creator of social wealth, also is main producers of the environment pollution, therefore, environmental pollution governance requires enterprises actively participate in. Enterprise full disclosure of environmental information, it is the precondition of environmental governance, is also the important way that investors understand company and important reference for investment decisions. Accounting information usually have certain information content and value for investors, and different types of information disclosure has a different influence on stock prices and stock trading volume (Wang, Y.T. et al, 2001). At the same time, the quality and level of the environmental information disclosure can reflect the corporate implementation of social responsibility. So, strengthen the environmental information disclosure research has important theoretical and practical significance. This article with all of the 2012 a-share listed companies as research samples, in-depth analysis of the influence of environmental information disclosure behavior on the firm future market value and investors' confidence in capital market. Aiming to promote the continuous improvement of environmental information disclosure and disclosure quality level, to meet the information needs of stakeholders, and promote the sustainable development of economy in china.

\section{Literature Review}

Foreign research on environmental information disclosure originated the social responsibility information disclosure in the 1940s, become a research hotspot after 70s. In the cause of the environmental information disclosure, previous scholars divided it into two aspects to study: companies choose active disclosure of environmental information disclosure and the interference factors. The causes of companies choose to take initiative and proactive to disclose environmental information are to avoid political cost, prevent reputational damage and fulfill social responsibility, etc. Maltby (2004) research shows that: the enterprise can show their special and important value through the environmental information disclosure. So enterprises actively improve its value to society not only response to the demand of the society. Interference factors of corporate disclosure of environmental information mainly includes the industry sensitivity (Comier et al, 2005; Frost, 2007), external control (Porteiro, 2008), company size, financial condition, debt levels, and corporate governance mechanism.

Domestic scholars have made the preliminary discussion on the market reaction and economic consequences of environmental information disclosure, but have not a unified direction. Shen 
H.T.(2010) theoretically discussed the market response and the economic consequences caused by the environmental information disclosure, and impact on the equity capital cost. Tang Y.J. \& X.S. Cheng(2005) empirical study found that the environmental information disclosure has a certain improvement, but not particularly significant impact on corporate performance and enterprise value. Tang, G.P. \&L.H. Li (2011) study found that the company environmental information disclosure is negatively related to the investors' confidence, environmental information disclosure index is negatively related to the value of the company.

Because Chinese scholar have not easy channel to achieve environmental information disclosure information, the environmental disclosure information data used by early study are collected manually. It is not sufficient that research of capital markets reaction for the environmental information disclosure, and there is still no exact significantly conclusions in China. Therefore, it is necessary to conduct a comprehensive in-depth research of all listed companies environmental information disclosure market reaction and economic consequences in China, in order to provide empirical evidence for improvement our country's environmental information disclosure.

\section{The Research Hypothesis and Research Model}

Theory Analysis. The theory, used to interpret environmental information disclosure market reaction, are mainly voluntary disclosure and signaling hypothesis, and modern behavior finance theory, etc.

(1) The voluntary disclosure and the signaling hypothesis. Voluntary disclosure theory regard that, although external pressure theory has certain reasonable and persuasive, but it does not explain enterprise voluntary disclosure of environmental information. This view think that inadequate disclosure of corporate information can discount enterprise investment value for the information users, Even list it in the worst, it would do more harm to the enterprise financing and the future development. Signal transmission is used to solve a series of problems caused by information asymmetry problem, Such as adverse selection problem. When a information is not timely disclosed, investors tend to think of it as a bad news. In order to avoid the occurrence of adverse selection problem, companies with "good news" more willing to take the initiative to disclose information, separate himself apart from the "bad news" enterprise (Dye, 1985; Verrecchia, 1983). Maltby(2004) research shows that the enterprise can show its special and important value through the social environment information disclosure. So enterprise is not only in social pressure to disclosure information, in order to show its not the worst, at the same time, in order to avoid the information user make a bad guess in the case of lack information, willing to take the initiative to open more information. In addition, the holding company's managers have incentive to signal transfer undervalue through voluntary disclosure, make their stock is worth more (Healy \& palequ, 2001).

(2) The modern behavioral finance theory. The modern behavioral finance theory, basis on the effective financial market theory, using cognitive psychology analysis investor behavior, is important theory to effective explain the investor behavior. The theory regard that human economic behavior under the influence of psychological factors, such as confidence, remorse, experiences, feelings, attitudes, opinions, attention and expectation, etc. Changing people's expectations and market decision-making behavior. Can be used to explain the investors market reaction for information disclosure. Chun wang, C. \& Xu, L.B.(2009), the Long Yuee(2010) argues that investor confidence, as investors' specific psychological phenomenon, will affect the company stock trading price and trading volume through the investor beliefs and behavior change, and thus affect the value of the company.

Research Hypothesis Put Forward. In today's increasingly prominent environmental problem in China, investors and other stakeholders have started to pay close attention to the environmental protection, Environmental information disclosure quality and disclosure level can reflect the corporate implementation of social environment responsibility. According to the modern behavioral finance theory, the company's disclosure of environmental information must have certain information content for investors, changing people's expectations and market decision-making behavior, affect stock market price and trading volume, and thus affect the company value. Therefore put forward: 
H1:Environmental information disclosure behavior are positive related to the added market value of listed companies.

$\mathrm{H} 2$ :Environmental information disclosure quality are positive related to the added market value of listed companies.

H3:Environmental information disclosure behavior can enhance investors confidence.

H4:High quality of environmental information disclosure can enhance investors confidence.

Research Model. Reference to previous research model, and according to the increase of variables this study, designed the following regression model to verify the above hypothesis:

$$
\begin{aligned}
& \triangle \mathrm{MVSit}+1=\beta 0+\beta 1 \mathrm{EDIit}+\beta 2 \text { Sizeit }+\beta 3 \text { Levit }+\sum \gamma \mathrm{jINDj}+\varepsilon \\
& \text { TurnMit }+1=\mathrm{a} 0+\mathrm{a} 1 \text { Discit }+\mathrm{a} 2 \text { Sizeit }+\mathrm{a} 3 \text { Levit }+\mathrm{a} 4 \mathrm{INSTit}+\mathrm{a} 5 \text { Lossit }+\Sigma \lambda \mathrm{jINDj}+\varepsilon \\
& \text { TurnMit+1=a0+a1EDIit+a2Sizeit+a3Levit+a4INSTit+a5BTMit+a6Lossit }+\Sigma \lambda j I N D j+\varepsilon \quad(\text { Model 3) }
\end{aligned}
$$

\begin{tabular}{|c|c|c|}
\hline variables & & variables definition \\
\hline Disc & $=$ & $\begin{array}{l}\text { Disclosure environmental information assignment } 1 \text {, otherwise the value } 0 . \\
\text { indicate companies disclose environmental information or not. }\end{array}$ \\
\hline $\mathrm{EDI}_{1}$ & $=$ & $\begin{array}{l}\text { Sum up environmental information disclosure entry score, reaction company } \\
\text { disclosure of environmental information content quantity. }\end{array}$ \\
\hline $\mathrm{EDI}_{2}$ & $=$ & $\begin{array}{l}\text { weighting value of each entries content(adopt } 0 、 0.01 、 0.02 \text { standard for } \\
\text { evaluation), reaction company disclosure of environmental information content } \\
\text { quality. }\end{array}$ \\
\hline MV & $=$ & The market value of listed companies, standardized by total assets. \\
\hline$\triangle \mathrm{MVS}$ & $=$ & $\begin{array}{l}\text { The stock market value of listed company value compared with the previous } \\
\text { quarter in the third quarter of } 2013 \text {, standardized by total assets. }\end{array}$ \\
\hline INST & $=$ & Proportion of institutional investor share holding \\
\hline BTM & $=$ & Book value to market value. \\
\hline & & Stock monthly turnover rate of jth month (Investor Confidence Index, $\mathrm{j}=1 \sim$ \\
\hline Turn $\mathrm{Mj}$ & $=$ & $\begin{array}{l}\text { 10) The } \mathrm{j} \text { month total trading volumes/ the total shares outstanding at the } \\
\text { beginning of the year }\end{array}$ \\
\hline Size & $=$ & natural logarithm of total assets \\
\hline Lev & $=$ & Debt level \\
\hline ID & $=$ & industry dummy variable \\
\hline $\mathrm{t}$ & $=$ & 2012 \\
\hline$\varepsilon$ & $=$ & error term \\
\hline
\end{tabular}

Variable Selection and Measurement. All the index and the index connotation explanation used in this paper listed in table 1 .

Table 1 Variables and variable definition

\section{The Sample Data}

Sample Selection Criteria and Data Sources. This paper selects 2012 a-share listed companies in the Shanghai and ShenZhen stock exchange as the overall sample, then, deletes the not complete data companies and extreme value companies, the final samples is 2098 companies. This study of financial data and market value index derived from the CSMAR database, The environmental information disclosure data is obtained by Manual sorting according to the "corporate social responsibility report" in CSMAR database; Institutional investors holding data taken from Wind database. 
Descriptive Statistics. Table 2 shows the descriptive statistics result of all samples. The mean of environmental information disclosure index Disc 、EDI1 EDI2 are 0.193, 0.0296, 0.0159 respectively in 2012. Proportion of disclosure environmental information companies is only $19.3 \%$, and there are larger differences in environmental information disclosure level between differences enterprises. The mean of $\triangle \mathrm{MV} 3$ is 0.2937 in the third quarter of 2013 , The mean of stock monthly turnover is 0.4775 , 0.2946, 0.4331, 0.4837, 0.4284, 0.4042 respectively in may, June, July August September, October, Shows that market turnover trend growing from June to August in 2013, Volatile 4-5 months, September to October showed a downward trend. That is to say, after the environmental information disclosure in April, the recent stock market active period is in the third quarter. It also suggests that the market influence of environmental information disclosure should be embodied in the third quarter in China market in 2013.

Table 2 All samples descriptive statistics

\begin{tabular}{lrrrr}
\hline \multicolumn{1}{c}{ variables } & mean & $\begin{array}{r}\text { standard } \\
\text { deviation }\end{array}$ & minimum & maximum \\
\hline Disc & 0.1930 & 0.3948 & 0.0000 & 1.0000 \\
EDI $_{1}$ & 0.0296 & 0.1003 & 0.0000 & 1.0000 \\
EDI $_{2}$ & 0.0159 & 0.0564 & 0.0000 & 1.0000 \\
$\triangle$ MV3 & 0.2937 & 0.6928 & -3.9149 & 16.2768 \\
TurnM 10 & 0.4042 & 0.3960 & 0.0002 & 4.3172 \\
TurnM 9 & 0.4284 & 0.4274 & 0.0001 & 6.0947 \\
TurnM 8 & 0.4837 & 0.4921 & 0.0002 & 6.4669 \\
TurnM 7 & 0.4331 & 0.4898 & 0.0030 & 5.1348 \\
TurnM 6 & 0.2946 & 0.3311 & 0.0022 & 4.8049 \\
TurnM 5 & 0.4775 & 0.4451 & 0.0001 & 4.5756 \\
TurnM 4 & 0.2806 & 0.3060 & 0.0015 & 3.3288 \\
Size & 22.0562 & 1.4798 & 15.7294 & 30.4956 \\
LEV & 0.4761 & 0.2334 & 0.0164 & 1.8973 \\
\hline
\end{tabular}

\section{Regression Results and Analysis}

Regression Results of Model1-H1,H2 be Verified. From table 3 can be seen: (1) The listed companies environmental information disclosure behavior index is significantly positive with the company's next seasonal added value at 5\% level. This suggests that the listed company disclosure of environmental information compared with the do not to disclose environmental information listed company, could improve the market value of listed companies. (2) The listed companies environmental information quantitative index and disclosure quality index are all significantly positive with the company's next seasonal added value at 5\% level. In short, Capital market response strongly to the environmental information disclosure. This from another Angle shows that environmental information disclosure quality of listed companies can more greatly improve the listed companies market value in the period of stock market active. Consistent with our expectations, hypothesis1, and 2 is verified. 
Table 3 Regression results of model 1

\begin{tabular}{|c|c|c|c|c|c|}
\hline & $\begin{array}{l}\triangle \mathrm{MV3} \\
\mathrm{t}(\mathrm{p})\end{array}$ & & $\begin{array}{l}\triangle \mathrm{MV3} \\
\mathrm{t}(\mathrm{p})\end{array}$ & & $\begin{array}{l}\triangle \mathrm{MV3} \\
\mathrm{t}(\mathrm{p})\end{array}$ \\
\hline \multirow[t]{2}{*}{ inter } & \multirow{2}{*}{$\begin{array}{c}3.923 * * * \\
(14.179)\end{array}$} & \multirow[t]{2}{*}{ inter } & $3.865 * * *$ & inter & $3.895 * * *$ \\
\hline & & & $(14.491)$ & & $(14.496)$ \\
\hline \multirow[t]{2}{*}{ Disc } & \multirow{2}{*}{$\begin{array}{c}0.085 * * \\
(2.092)\end{array}$} & \multirow[t]{2}{*}{$\mathrm{EDI}_{1}$} & $0.566^{* *}$ & $\mathrm{EDI}_{2}$ & $0.352 * *$ \\
\hline & & & $(2.095)$ & & $(2.284)$ \\
\hline \multirow[t]{2}{*}{ Size } & \multirow{2}{*}{$\begin{array}{l}-0.15 * * * \\
(-11.693)\end{array}$} & \multirow[t]{2}{*}{ Size } & $-0.147 * * *$ & Size & $-0.148 * * *$ \\
\hline & & & $(-11.959)$ & & $(-11.982)$ \\
\hline \multirow[t]{2}{*}{ LEV } & \multirow{2}{*}{$\begin{array}{c}0.046 \\
(0.663)\end{array}$} & \multirow[t]{2}{*}{$\mathrm{LEV}$} & 0.04 & LEV & 0.039 \\
\hline & & & $(0.576)$ & & $(0.569)$ \\
\hline IND & Control & IND & Control & IND & Control \\
\hline $\operatorname{Adj} R^{2}$ & \multicolumn{2}{|c|}{0.131} & \multicolumn{2}{|c|}{0.131} & 0.131 \\
\hline $\mathrm{F}(\mathrm{P})$ & \multicolumn{2}{|c|}{$19.585(.000)$} & \multicolumn{2}{|c|}{$19.586(.000)$} & $19.643(.000)$ \\
\hline MaxVIF & \multicolumn{2}{|c|}{1.655} & \multicolumn{2}{|c|}{1.655} & 1.655 \\
\hline $\mathrm{N}$ & \multicolumn{2}{|c|}{2098} & \multicolumn{2}{|c|}{2098} & 2098 \\
\hline
\end{tabular}

Note:1) Model: $\triangle$ MVS it $+1=\beta 0+\beta 1$ EDI it $+\beta 2$ Sizeit $+\beta 3$ Levit $+\Sigma \gamma j$ INDj $+\varepsilon ; 2)$ The definition of all variables are shown in table $1 ; 3)^{* * *}, * *$ and $*$ is $1 \%, 5 \%$ and $10 \%$ significant level.

Regression Results of Model2-H3 Cannot Be Verified. From regression results of model 2 table 4 can be seen: The listed companies environmental information disclosure behavior index is not significantly positive with the company's stock monthly turnover rate. This suggests that there was no significant difference between the listed company disclosure of environmental information and do not disclose. Hypothesis 3 cannot be verified.

Regression Results of Model 2 - H4 Be Verified. From regression results of model 3 table 5 and table 6 can be seen: (1) The listed companies environmental information disclosure quantitative index and disclosure quality index are all significantly positive with the company's may, June, July, August stock monthly turnover rate. Especially during the stock market "active" period, July and August, the significance level reached $5 \%$ and $1 \%$. This suggests that environmental information disclosure quantitative and quality can significantly increase the stock turnover rate, and improve investor confidence. This confidence with our anticipation, Hypothesis 4 is verified.

Table 4 Regression results of model 2

\begin{tabular}{|c|c|c|c|c|c|c|}
\hline & $\begin{array}{c}\text { Turn M } 5 \\
t(p)\end{array}$ & $\begin{array}{l}\text { TurnM6 } \\
\mathrm{t}(\mathrm{p})\end{array}$ & $\begin{array}{l}\text { TurnM7 } \\
\mathrm{t}(\mathrm{p})\end{array}$ & $\begin{array}{c}\text { Turn M8 } \\
\mathrm{t}(\mathrm{p})\end{array}$ & $\begin{array}{c}\text { Turn M9 } \\
\mathrm{t}(\mathrm{p})\end{array}$ & $\begin{array}{c}\text { Turn M } 10 \\
t(p)\end{array}$ \\
\hline constant & $\begin{array}{l}2.084 * * * \\
(11.676)\end{array}$ & $\begin{array}{l}1.344 * * * \\
(9.808)\end{array}$ & $\begin{array}{l}2.038 * * * \\
(10.406)\end{array}$ & $\begin{array}{l}2.266 * * * \\
(11.564)\end{array}$ & $\begin{array}{l}1.714 * * * \\
(9.795)\end{array}$ & $\begin{array}{l}1.510 * * * \\
(9.366)\end{array}$ \\
\hline Disc & $\begin{array}{l}-.031 \\
(-1.190) \\
\end{array}$ & $\begin{array}{l}-.018 \\
(-.893) \\
\end{array}$ & $\begin{array}{l}-.020 \\
(-.691) \\
\end{array}$ & $\begin{array}{l}-.042 \\
(-1.478) \\
\end{array}$ & $\begin{array}{l}-.022 \\
(-.878) \\
\end{array}$ & $\begin{array}{l}-.036 \\
(-1.570) \\
\end{array}$ \\
\hline Size & $\begin{array}{l}.064 * * * \\
(-7.603)\end{array}$ & $\begin{array}{l}-.037 * * * \\
(-5.747) \\
\end{array}$ & $\begin{array}{l}-.049 * * * \\
(-5.400)\end{array}$ & $\begin{array}{l}-.065 * * * \\
(-7.056)\end{array}$ & $\begin{array}{l}-.042 * * * \\
(-5.172)\end{array}$ & $\begin{array}{l}.036 * * * \\
(-4.79)\end{array}$ \\
\hline LEV & $\begin{array}{l}-.204 * * * \\
(-4.529)\end{array}$ & $\begin{array}{l}-.158 * * * \\
(-4.595)\end{array}$ & $\begin{array}{l}-.306 * * * \\
(-6.206)\end{array}$ & $\begin{array}{l}-.231 * * * \\
(-4.665)\end{array}$ & $\begin{array}{l}-.136 * * * \\
(-3.079)\end{array}$ & $\begin{array}{l}-.196 * * * \\
(-4.844)\end{array}$ \\
\hline INST & $\begin{array}{l}-.305 * * * \\
(-8.462)\end{array}$ & $\begin{array}{l}-.208 * * * \\
(-7.511)\end{array}$ & $\begin{array}{l}-.307 * * * \\
(-7.778)\end{array}$ & $\begin{array}{l}-.342 * * * \\
(-8.602)\end{array}$ & $\begin{array}{l}-.321 * * * \\
(-9.020)\end{array}$ & $\begin{array}{l}-.298 * * * \\
(-9.132)\end{array}$ \\
\hline loss & $\begin{array}{l}-.103 * * * \\
(-3.311)\end{array}$ & $\begin{array}{l}-.036 \\
(-1.501)\end{array}$ & $\begin{array}{l}-.087 * * \\
(-2.555)\end{array}$ & $\begin{array}{l}-.108 * * * \\
(-3.153)\end{array}$ & $\begin{array}{l}-.070 * * \\
(-2.283)\end{array}$ & $\begin{array}{l}-.066 * * \\
(-2.368)\end{array}$ \\
\hline $\mathrm{R} 2$ & & 77 & 32 & .181 & .135 & 152 \\
\hline $\mathrm{F}(\mathrm{p})$ & $24.397(0.000)$ & $17.446(0.000)$ & $21.568(0.000)$ & $24.930(0.000)$ & $17.880(0.000)$ & ) $20.343(0.000)$ \\
\hline Max VIF & 1.938 & 1.932 & 1.927 & 1.918 & 1.921 & 1.929 \\
\hline $\mathrm{N}$ & 2098 & 2098 & 2098 & 2098 & 2098 & 2098 \\
\hline
\end{tabular}


Note: 1) Model: TurnMit+1= a0+a1Discit++ a2 Sizeit+a3Levit $+a 4$ INSTit+a5Lossit $+\Sigma \lambda j$ INDj $+\varepsilon ; 2$ ) The definition of all variables are shown in table $1 ; 3)^{* * *}$, ** and $*$ is $1 \%, 5 \%$ and $10 \%$ significant level.

Table 5 Regression results of model 3(1)

\begin{tabular}{|c|c|c|c|c|c|c|c|}
\hline & $\begin{array}{l}\text { Turn M4 } \\
\mathrm{t}(\mathrm{p})\end{array}$ & $\begin{array}{l}\text { Turn M } 5 \\
t(p)\end{array}$ & $\begin{array}{l}\text { TurnM6 } \\
\mathrm{t}(\mathrm{p})\end{array}$ & $\begin{array}{l}\text { TurnM7 } \\
t(p)\end{array}$ & $\begin{array}{l}\text { Turn M8 } \\
\mathrm{t}(\mathrm{p})\end{array}$ & $\begin{array}{l}\text { TurnM9 } \\
t(p)\end{array}$ & $\begin{array}{l}\text { TurnM10 } \\
\mathrm{t}(\mathrm{p})\end{array}$ \\
\hline constant & $\begin{array}{l}1.63^{*} \\
(7.118)\end{array}$ & $\begin{array}{l}2.766 \\
(8.535)\end{array}$ & $\begin{array}{l}1.458 \\
(7.71)\end{array}$ & $\begin{array}{l}2.375 \\
(7.483)\end{array}$ & $\begin{array}{l}2.376 \\
9.305\end{array}$ & $\begin{array}{l}2.057^{3} \\
(7.945)\end{array}$ & $\begin{array}{l}1.619^{\text {(6.*.* }} \\
(6.03)\end{array}$ \\
\hline $\mathrm{EDI}_{1}$ & $\begin{array}{l}0.134 \\
(1.19)\end{array}$ & $\begin{array}{l}0.281^{*} \\
1.771\end{array}$ & $\begin{array}{l}0.159^{*} \\
(1.722)\end{array}$ & $\begin{array}{l}0.318 \\
(2.072)\end{array}$ & $\begin{array}{l}0.339 \\
2.731\end{array}$ & $\begin{array}{l}0.136 \\
(1.08)\end{array}$ & $\begin{array}{l}0.128 \\
(0.982)\end{array}$ \\
\hline Size & $\begin{array}{l}-0.068^{\text {*.*.* }} \\
(-6.257)\end{array}$ & $\begin{array}{l}-0.116 \\
-7.525\end{array}$ & $\begin{array}{c}-0.056^{\text {***** }} \\
(-6.231)\end{array}$ & $\begin{array}{l}-0.088^{2,9 x^{2}} \\
-5.781\end{array}$ & $\begin{array}{l}-0.09^{* * * *} \\
-7.446\end{array}$ & $\begin{array}{l}-0.071^{\text {*as: }} \\
(-5.798)\end{array}$ & $\begin{array}{l}-0.059^{\text {. }} \\
(-4.659)\end{array}$ \\
\hline LEV & $\begin{array}{l}0.056 \\
(0.715)\end{array}$ & $\begin{array}{l}0.08 \\
(0.72)\end{array}$ & $\begin{array}{l}-0.018 \\
(-0.281)\end{array}$ & $\begin{array}{l}-0.05 \\
(-0.456)\end{array}$ & $\begin{array}{l}0.036 \\
(0.42)\end{array}$ & $\begin{array}{l}-0.121 \\
(-1.372)\end{array}$ & $\begin{array}{l}-0.038 \\
(-0.417)\end{array}$ \\
\hline INST & $\begin{array}{l}-0.063 \\
(-1.424)\end{array}$ & $\begin{array}{l}-0.147 \\
(-2.343)\end{array}$ & $\begin{array}{l}-0.076 \\
(-2.076)\end{array}$ & $\begin{array}{l}-0.158 \\
(-2.566)\end{array}$ & $\begin{array}{l}-0.167 \\
(-3.394)\end{array}$ & $\begin{array}{l}-0.129^{n+1} \\
(-2.578)\end{array}$ & $\begin{array}{l}-0.133^{3} \\
(-2.563)\end{array}$ \\
\hline BTM & $\begin{array}{l}0.119^{\text {s.9.96 }} \\
(3.616)\end{array}$ & $\begin{array}{l}0.222^{*}, * * * \\
(4.782)\end{array}$ & $\begin{array}{c}0.089^{\text {*.F.* }} \\
(3.279)\end{array}$ & $\begin{array}{l}0.09^{\text {*** }} \\
(1.989)\end{array}$ & $\begin{array}{l}0.103^{\text {F*:. }} \\
(2.822)\end{array}$ & $\begin{array}{l}0.124^{\text {*.*.*2 }} \\
(3.373)\end{array}$ & $\begin{array}{l}0.091^{* * *} \\
(2.388)\end{array}$ \\
\hline loss & $\begin{array}{l}-0.076^{*} \\
(-1.905)\end{array}$ & $\begin{array}{l}-0.078 \\
(-1.38)\end{array}$ & $\begin{array}{l}-0.025 \\
(-0.756)\end{array}$ & $\begin{array}{l}-0.071 \\
(-1.289)\end{array}$ & $\begin{array}{l}-0.051 \\
(-1.155)\end{array}$ & $\begin{array}{l}-0.059 \\
(-1.308)\end{array}$ & $\begin{array}{l}-0.086 \\
(-1.829)\end{array}$ \\
\hline IND & control & control & control & control & control & control & control \\
\hline $\operatorname{Adj} R^{2}$ & 0.191 & 0.274 & 0.212 & 0.232 & 0.311 & 0.412 & 0.375 \\
\hline $\mathrm{F}$ (sig.) & $5.72^{*}$ & $8.563^{*}$ & $6.342^{\prime \prime}$ & $6.977^{* 1}$ & $10.093^{*}$ & $14.932^{*}$ & 12.958 \\
\hline Max VIF & 3.36 & 3.36 & 3.36 & 3.36 & 3.36 & 3.36 & 3.36 \\
\hline $\mathrm{N}$ & 405 & 405 & 405 & 405 & 405 & 405 & 405 \\
\hline
\end{tabular}

Note: 1) Model: TurnMit+1 = a0+a1EDIit++ a2 Sizeit+a3Levit +a4INSTit +a5BTMit +a6Lossit + $\Sigma \lambda \mathrm{jINDj}+\varepsilon ; 3)^{* * *}, * *$ and $*$ is $1 \%, 5 \%$ and $10 \%$ significant level.

Table 6 Regression results of model 3(2)

\begin{tabular}{|c|c|c|c|c|c|c|c|}
\hline & $\begin{array}{l}\text { TurnM4 } \\
\mathrm{t}(\mathrm{p})\end{array}$ & $\begin{array}{l}\text { Turn M5 } \\
\mathrm{t}(\mathrm{p})\end{array}$ & $\begin{array}{l}\text { TurnM6 } \\
\mathrm{t}(\mathrm{p})\end{array}$ & $\begin{array}{l}\text { TurnM7 } \\
\mathrm{t}(\mathrm{p})\end{array}$ & $\begin{array}{l}\text { TurnM8 } \\
\mathrm{t}(\mathrm{p})\end{array}$ & $\begin{array}{l}\text { TurnM9 } \\
\mathrm{t}(\mathrm{p})\end{array}$ & $\begin{array}{l}\text { TurnM10 } \\
\mathrm{t}(\mathrm{p})\end{array}$ \\
\hline constant & $\begin{array}{l}1.645^{3} \\
7.173\end{array}$ & $\begin{array}{l}2.787^{7 . \cdots+1} \\
8.592\end{array}$ & $\begin{array}{l}1.464^{2} \\
7.726\end{array}$ & $\begin{array}{l}2.388 \\
7.509\end{array}$ & $\begin{array}{l}2.379 \\
9.286\end{array}$ & $\begin{array}{l}2.06 \\
7.941\end{array}$ & $\begin{array}{l}1.611 \\
5.984\end{array}$ \\
\hline $\mathrm{EDI}_{2}$ & $\begin{array}{l}0.097 \\
1.466\end{array}$ & $\begin{array}{l}0.188^{* *} \\
2.017\end{array}$ & $\begin{array}{l}0.095^{*} \\
1.766\end{array}$ & $\begin{array}{l}0.192^{* *} \\
2.14\end{array}$ & $\begin{array}{l}0.187^{\text {P. }} \\
2.576\end{array}$ & $\begin{array}{l}0.079 \\
1.074\end{array}$ & $\begin{array}{l}0.056 \\
0.732\end{array}$ \\
\hline Size & $\begin{array}{l}-0.069^{\text {. }} \\
-6.315\end{array}$ & $\begin{array}{l}-0.117^{*} \\
-7.584\end{array}$ & $\begin{array}{l}-0.056^{\text {क* }} \\
-6.249\end{array}$ & $\begin{array}{l}-0.088^{* 3 *} \\
-5.809\end{array}$ & $\begin{array}{l}-0.091 \\
-7.429\end{array}$ & $\begin{array}{l}-0.072^{\text {. }} \\
-5.797\end{array}$ & $\begin{array}{l}-0.059^{3} \\
-4.612\end{array}$ \\
\hline LEV & $\begin{array}{l}0.053 \\
0.676\end{array}$ & $\begin{array}{l}0.074 \\
0.671\end{array}$ & $\begin{array}{l}-0.021 \\
-0.318\end{array}$ & $\begin{array}{l}-0.055 \\
-0.503\end{array}$ & $\begin{array}{l}0.032 \\
0.374\end{array}$ & $\begin{array}{l}-0.122 \\
-1.392\end{array}$ & $\begin{array}{l}-0.039 \\
-0.424\end{array}$ \\
\hline INST & $\begin{array}{l}-0.064 \\
-1.44\end{array}$ & $\begin{array}{l}-0.148 \\
-2.362\end{array}$ & $\begin{array}{l}-0.077^{\text {nt }} \\
-2.086\end{array}$ & $\begin{array}{l}-0.158^{* \pi} \\
-2.579\end{array}$ & $\begin{array}{l}-0.168 \\
-3.401\end{array}$ & $\begin{array}{l}-0.129 \\
-2.584\end{array}$ & $\begin{array}{l}-0.133^{n+1} \\
-2.559\end{array}$ \\
\hline BTM & $\begin{array}{l}0.12^{\text {**F }} \\
3.643\end{array}$ & $\begin{array}{l}0.223^{\text {*as }} \\
4.812\end{array}$ & $\begin{array}{l}0.089^{* * *} \\
3.296\end{array}$ & $\begin{array}{l}0.091^{*} \\
2.012\end{array}$ & $\begin{array}{l}0.103^{\text {* }} \\
2.833\end{array}$ & $\begin{array}{l}0.125^{\text {5.9.9. }} \\
3.381\end{array}$ & $\begin{array}{l}0.091^{*} \\
2.38\end{array}$ \\
\hline loss & $\begin{array}{l}-0.077^{*} \\
-1.92\end{array}$ & $\begin{array}{l}-0.079 \\
-1.398\end{array}$ & $\begin{array}{l}-0.025 \\
-0.769\end{array}$ & $\begin{array}{l}-0.072 \\
-1.302\end{array}$ & $\begin{array}{l}-0.052 \\
-1.173\end{array}$ & $\begin{array}{l}-0.059 \\
-1.316\end{array}$ & $\begin{array}{l}-0.086 \\
-1.834^{*}\end{array}$ \\
\hline IND & Control & Control & Control & Control & Control & Control & Control \\
\hline $\operatorname{Adj} R^{2}$ & 0.192 & 0.276 & 0.212 & 0.232 & 0.31 & 0.412 & 0.374 \\
\hline$F($ sig. $)$ & $5.767^{* * * * 4}$ & $8.63^{\text {**** }}$ & $6.352^{\text {*.:F* }}$ & $6.996^{* * * * 1 \times}$ & $10.031^{\text {* }}$ & $14.93^{\text {**** }}$ & $12.922^{* * * * *}$ \\
\hline Max VIF & 3.371 & 3.371 & 3.371 & 3.371 & 3.371 & 3.371 & 3.371 \\
\hline $\mathrm{N}$ & 405 & 405 & 405 & 405 & 405 & 405 & 405 \\
\hline
\end{tabular}

Note:1) Model: TurnMit+1 = a0+a1EDIit++ a2 Sizeit+a3Levit +a4INSTit +a5BTMit +a6Lossit + $\Sigma \lambda \mathrm{jINDj}+\varepsilon ; 3)^{* * *}, * *$ and ${ }^{*}$ is $1 \%, 5 \%$ and $10 \%$ significant level. 


\section{Research Conclusions and Contributions}

This paper inspects market reactions of environmental information disclosure. The research gain the following conclusions: (1)The listed companies` environmental information disclosure behavior index and environmental information disclosure quality index both are significantly positive with the company's next seasonal added value.; (2)The disclosure quality significantly positive correlation with investor confidence.

In short, the higher quality of disclosure of environmental information can higher significantly improve the company value and investors' confidence in Chinese capital market. Indicate that environmental information disclosure has significantly increased the value of the company. Capital market response strongly to the environmental information disclosure. Environmental information disclosure behavior and disclosure quality played the role of market mechanism, and exerted positive influence on investors decision-making and company's market value in a certain extent, which is beneficial to promote enterprise voluntary disclosing environmental information.

\section{References}

[1] Gary. A new ideological of western accounting theory in the $90 \mathrm{~s}$ - green accounting theory, Accounting Research, 5 (1992)23-28.

[2] J.X. Geng, R.J. Jiao, Primary exploration of environmental accounting information disclosure of listed companies. Accounting Research, 1( 2002)43-47.

[3] J.F. Li, Our country enterprise environment report: the present situation, demand and future, Journal of accounting research, 1(2002)42-50.

[4] H.T. Shen, X.L. Li, The analysis of environmental information disclosure of listed companies of heavy pollution industry in China, Journal of securities market leader, 6 (2010)51-57.

[5] J.Y. Shi, X.T. Sun, F.F. Liu, Investors overconfidence and corporate investment behavior. Journal of northeastern university, 1(2011) 33-37.

[6] Y.J. Tang, X.S. Cheng, Information disclosure mechanism evaluation, information disclosure index and enterprise performance -- based on the survey of 931 listed companies. Journal of management review, 10(2005)8-15.

[7] Y. T. Wang, Z. Sun, S.M. Chen, Accounting reform and the quality of accounting information-empirical evidence from China's securities market. Journal of accounting research, 7 (2001)16-26. 